\title{
Avaliação das Perdas Endógenas e das Exigências de Fósforo, por Meio da Técnica da Diluição Isotópica, para Suínos em Crescimento ${ }^{1}$
}

\author{
João Batista Lopes², Dorinha Miriam Silber S. Vitti ${ }^{3}$, Agustinho Valente de Figueirêdo², Hacy \\ Pinto Barbosa 4
}

\begin{abstract}
RESUMO - Esta pesquisa foi realizada para determinar as perdas endógenas mínimas, as exigências nutricionais de fósforo (P) para mantença, a biodisponibilidade do P de fosfato bicálcico e a variação da concentração de P no plasma, em suínos de 20 kg PV. Os suínos foram alimentados com rações contendo diferentes níveis de $\mathrm{P}$ total $(0,30 ; 0,40 ; 0,51 ; 0,65 ; \mathrm{e} 0,73 \%)$. Vinte leitões híbridos comerciais, machos, castrados, foram alojados em gaiolas metabólicas por um período de sete dias de adaptação e sete dias de coleta total de fezes, urina e amostras de sangue. No primeiro dia da fase de coleta, cada animal foi injetado, por via endovenosa, com $7,4 \mathrm{MBq}$ de ${ }^{32} \mathrm{P}$, como traçador. Os valores de P endógeno fecal foram de 8,59; 8,43; 9,81; 11,28; e 12,75 mg P/kg PV•dia; P retido, 25,68; 67,05; 108,29; 131,56; e 135,88 mg P/kg PV•dia; e P absorvido, 34,55; 75,93; 124,41; 162,97; e 175,00 mg P/kg PV•dia, respectivamente, para as dietas A, B, C, D e E. A perda endógena fecal mínima dos leitões foi de $23,92 \mathrm{mg} \mathrm{P} / \mathrm{kg}$ PV•dia e a exigência diária de P para mantença de leitões, em dietas suplementadas com fosfato bicálcico, de $38,55 \mathrm{mg} / \mathrm{kg}$ PV·dia. A disponibilidade biológica de P do fosfato bicálcico foi de $90,30 \%$.
\end{abstract}

Palavras-chave: rádiofósforo, suíno, nutrição, absorção real

\section{Evaluation of Endogenous Fecal Losses and of Phosphorus Requirements for Swines in the Growing Phase Based on Isotopic Dilution Technique}

\begin{abstract}
This research was conducted to determine the minimum endogenous losses, the nutritional requirement of phosphorus $(\mathrm{P})$ for maintenance, the biological availability of $\mathrm{P}$ from dicalcium phosphate and the variation of plasma $\mathrm{P}$ concentration in swine of $20 \mathrm{~kg} \mathrm{LW}$. The pigs were fed diets containing different levels of total phosphorus (.30, .40, .51, .65 and .73\%). Twenty commercial crossbred barrows were maintained in metabolic cages for a seven-day period of adaptation and seven days of total collection of feces, urine and blood samples. In the first day of the collection period, each animal was injected intravenously with $7.4 \mathrm{MBq}$ of ${ }^{32} \mathrm{P}$ as metabolic tracer. The values of endogenous fecal $\mathrm{P}$ were of $8.59,8.43,9.81,11.28$, and $12.75 \mathrm{mg} \mathrm{P} / \mathrm{kg} \mathrm{LW} \bullet \mathrm{day}$, of P retained were $25.68,67.05,108.29,131.56$, and $135.88 \mathrm{mg} \mathrm{P} / \mathrm{kg} \mathrm{LW} \bullet$ day, and of absorption P were 34.55, 75.93, 124.41, 162.97, and 175.00 mg P/ $\mathrm{kg} \mathrm{LW} \bullet$ day, respectively, for diets A, B, C, D and E. The minimum endogenous fecal loss was $23.92 \mathrm{mg} / \mathrm{kg} \mathrm{LW} \bullet$ day and the daily P requirement for maintenance of pigs, in diets supplemented with dicalcium phosphate, was $38.58 \mathrm{mg} \mathrm{P} / \mathrm{kg} \mathrm{LW \bullet day.} \mathrm{The} \mathrm{biological}$ availability of $\mathrm{P}$ from dicalcium phosphate was $90.30 \%$.
\end{abstract}

Key words: radiophosphorus, swine, nutrition, true absorption

\section{Introdução}

Os estudos sobre digestibilidade e biodisponibilidade do fósforo em dietas de suínos têm apresentado grande variação nos resultados, propiciada não apenas pelo crescente desenvolvimento e produtividade animal, mas também pela diversificação das técnicas usadas para avaliação desse importante nutriente. Neste sentido, a indústria de rações necessita de informações precisas sobre a disponibilidade biológica do fósforo, para produzir dietas que atendam às exigências dos animais com menor custo (CUPÁK et al., 1972).
A suplementação adequada do fósforo nas rações, além de evitar gastos desnecessários decorrentes de desperdícios, pode contribuir para melhor desempenho do rebanho, caracterizado por taxas de crescimento mais rápidas e eficientes, bem como pelo adequado desenvolvimento dos ossos. Além disso, pode proporcionar economia tanto para produtores como para os fabricantes de ração e reduzir, no meio ambiente, a descarga poluente do fósforo, que acompanha os dejetos dos animais (CAVALCANTE, 1994). Dessa forma, o conhecimento da absorção real desse nutriente poderá contribuir para determina-

\footnotetext{
1 Pesquisa financiada pela FAPESP e parte da tese do primeiro autor.

2 Professores adjuntos do Departamento de Zootecnia do CCA-UFPI.

${ }^{3}$ Professor do Centro de Energia Nuclear na Agricultura da USP.

${ }^{4}$ Pesquisador do Instituto de Zootecnia de Nova Odessa.
} 
ção das exigências nutricionais e de sua utilização de modo racional.

Para a avaliação da disponibilidade biológica do fósforo, é necessária a determinação das suas perdas por meio das secreções endógenas fecal e urinária, uma vez que essas perdas endógenas são parte integrante da avaliação biológica do alimento e devem ser incorporadas na avaliação das exigências nutricionais (AKINSOYINN, 1986; FERNANDEZ, 1995b). Entre as várias técnicas para determinação da disponibilidade biológica do $\mathrm{P}$, o uso de radioisótopos pode se sobressair em estudos destinados a medir absorção real e distribuição do fósforo nos tecidos (VITTI, 1989).

Neste contexto, idealizou-se um estudo para determinar as perdas endógenas mínimas, as exigências de fósforo para mantença, a disponibilidade biológica do $\mathrm{P}$ do fosfato bicálcico e a variação do conteúdo de $\mathrm{P}$ no plasma, em suínos no início da fase de crescimento, em dietas contendo diferentes níveis de fósforo.

\section{Material e Métodos}

A pesquisa foi desenvolvida no Centro de Energia Nuclear na Agricultura da Universidade de São Paulo, utilizando-se 20 leitões híbridos comerciais, machos, castrados, com peso médio de $20 \mathrm{~kg}$.

$\mathrm{O}$ delineamento experimental foi o de blocos ao acaso, com cinco tratamentos e quatro repetições. Os resultados foram submetidos à análise de regressão, relacionando-os com o P consumido (SAS, 1986).

Os tratamentos consistiram de rações formulada à base de milho, farelo de soja e suplemento vitamínico e mineral, contendo diferentes níveis de fósforo: A: $0,30 \%$ de fósforo total $(\mathrm{Pt})$, provenientes dos ingredientes de origem vegetal; B: 0,40; C: 0,51; D: 0,65; e E: $0,73 \%$ Pt. O ajuste do fósforo total, nas dietas B, $\mathrm{C}, \mathrm{D}$, e E, foi obtido pela adição de fosfato bicálcico.

A comparação das dietas experimentais pode ser visualizada na Tabela 1 .

Os leitões foram alojados em gaiolas metabólicas

Tabela 1 - Composição das dietas experimentais

Table 1 - Composition of the experimental diets

\begin{tabular}{|c|c|c|c|c|c|}
\hline \multirow{3}{*}{$\begin{array}{l}\text { Ingrediente } \\
\text { Ingredient }\end{array}$} & \multicolumn{5}{|c|}{$\begin{array}{l}\text { Nível de fósforo total (\%) } \\
\text { Level of total phosphorus }\end{array}$} \\
\hline & $\mathrm{A}$ & $\mathrm{B}$ & $\mathrm{C}$ & $\mathrm{D}$ & $\mathrm{E}$ \\
\hline & $(0,30)$ & $(0,40)$ & $(0,51)$ & $(0,65)$ & $(0,73)$ \\
\hline$\overline{\text { Milho (kg) }}$ & 84,80 & 83,46 & 82,17 & 81,86 & 82,14 \\
\hline Corn & & & & & \\
\hline Farelo soja (kg) & 12,14 & 13,25 & 14,28 & 14,29 & 13,58 \\
\hline Soybean meal & & & & & \\
\hline Óleo soja $(\mathrm{kg})$ & 0,50 & 0,60 & 0,70 & 0,80 & 1,10 \\
\hline $\begin{array}{l}\text { Soybean oil } \\
\text { Calcário }^{1}(\mathrm{~kg})\end{array}$ & 1,66 & 1,27 & 0,90 & 0,40 & 0,10 \\
\hline Limestone & & & & & \\
\hline $\begin{array}{l}\text { P bicálcico }{ }^{2}(\mathrm{~kg}) \\
\text { Dicalcium } P\end{array}$ & 0,00 & 0,52 & 1,05 & 1,75 & 2,18 \\
\hline $\mathrm{Sal}(\mathrm{kg})$ & 0,40 & 0,40 & 0,40 & 0,40 & 0,40 \\
\hline $\begin{array}{l}\text { Salt } \\
\text { Suplemento mineral }(\mathrm{kg})^{3}\end{array}$ & 0,10 & 0,10 & 0,10 & 0,10 & 0,10 \\
\hline $\begin{array}{l}\text { Mineral supplement } \\
\text { Suplemento vitamínico }(\mathrm{kg})^{3}\end{array}$ & 0,40 & 0,40 & 0,40 & 0,40 & 0,40 \\
\hline $\begin{array}{l}\text { Vitamin supplement } \\
\text { Proteína bruta }(\%)\end{array}$ & 13,37 & 13,76 & 14,11 & 14,09 & 13,79 \\
\hline $\begin{array}{l}\text { Crude protein } \\
\text { Energia digestível ( } \mathrm{kcal} / \mathrm{kg})^{4} \\
\text { Digestible energy }\end{array}$ & 3.412 & 3.411 & 3.408 & 3.406 & 3.415 \\
\hline $\begin{array}{l}\text { P total }(\%) \\
\text { Total } P\end{array}$ & 0,30 & 0,40 & 0,51 & 0,65 & 0,73 \\
\hline $\mathrm{Ca}: \mathrm{P}$ & 2,00 & 1,50 & 1,18 & 0,92 & 0,82 \\
\hline $\begin{array}{l}{ }^{1} \mathrm{Ca}(\%)=32,3 . \\
2 \mathrm{Ca}(\%)=23,13 \%, \mathrm{P}(\%)=19,9 \\
{ }^{3} \text { Quantidade/kg de mistura }(\mathrm{Amou} \\
750 \mathrm{mg} ; \text { Vit } \mathrm{B}_{6}, 250 \mathrm{mg} ; \text { Vit } \mathrm{B}_{12}, 3 \\
\text { crescimento (growth promoter), } 12 \\
\text { I, } 1000 \mathrm{mg} ; \text { Se, } 120 \mathrm{mg} .\end{array}$ & $\begin{array}{l}\text { ): Vit A, } \\
\text { na (niacin } \\
\text { oxidante }\end{array}$ & $\begin{array}{l}\text { I; Vit } D_{3}, \\
\text { Ác. pantc } \\
3750 \mathrm{~m}\end{array}$ & $\begin{array}{l}\text { it } \mathrm{E}, 3.0 \\
\text { enic acic } \\
\mathrm{ng} ; \mathrm{Cu} \text {, }\end{array}$ & $\begin{array}{l}00 \mathrm{mg} ; \\
\text { ha (cholit } \\
70.000\end{array}$ & $\begin{array}{l}\mathrm{mg} ; \text { Vit } \\
\text {; promo } \\
0.000 \mathrm{~m}\end{array}$ \\
\hline
\end{tabular}


por um período de sete dias de adaptação e sete de coleta total de fezes, urina e amostras de sangue, sendo um décimo do conteúdo das fezes e da urina armazenado em congelador para as devidas análises. No primeiro dia da fase de coleta, foi introduzido, como traçador, o ${ }^{32} \mathrm{P}$ por via endovenosa, na dose de $7,4 \mathrm{MBq}$, como fosfato de sódio $\left(\mathrm{Na}_{2} \mathrm{HPO}_{4}\right)$, livre de carregador.

Os animais receberam diariamente duas refeições, com base no tamanho metabólico, levando-se em consideração o menor consumo observado na fase pré-experimental, entre os animais de cada bloco. A água foi fornecida à vontade.

A análise bromatológica das dietas foi realizada conforme as recomendações da AOAC (1980) e a determinação do cálcio, por espectrometria de absorção atômica (ZAGATTO et al., 1979). A concentração de fósforo inorgânico no plasma e na urina foi avaliada por colorimetria (FISKE e SUBBAROW, 1925) e nas fezes e nas dietas, pelo método vanadato-molibdato (SARRUGE e HAAG, 1974). A contagem da atividade do ${ }^{32} \mathrm{P}$ foi realizada por efeito Cerenkov (IAEA, 1979; NASCIMENTO FILHO e LOBÃO, 1977).

Os cálculos do percentual da atividade injetada do ${ }^{32} \mathrm{P}\left(\mathrm{AI}{ }^{32} \mathrm{P}\right)$ encontrada no plasma e nas fezes e as atividades específicas (AE) no plasma e nas fezes foram feitos de acordo com LOFGREN (1960), ao passo que o valor do fósforo endógeno se baseou em COMAR et al. (1953) e VITTI (1989):

$\mathrm{AI}{ }^{32} \mathrm{P}=[$ (contagem por minuto de $1 \mathrm{~mL}$ de plasma ou $1 \mathrm{~g}$ de fezes/contagem por minuto de uma solução radioativa padrão) x 100];

$\mathrm{AE}={ }^{32} \mathrm{P} / \mathrm{P}$ total;

$\% \mathrm{P}$ endógeno fezes $=$ (atividade específica do ${ }^{32} \mathrm{P}$ fezes/atividade específica ${ }^{32} \mathrm{P}$ plasma) $\mathrm{x} 100$; e

$\mathrm{P}$ endógeno total nas fezes $=\% \mathrm{P}$ endógeno fezes $\mathrm{x} P$ total excretado nas fezes.

A absorção real do fósforo foi calculada considerando-se o $\mathrm{P}$ consumido, o excretado nas fezes e na urina e o endógeno (GRACE, 1981; VITTI, 1989; FERNANDEZ, 1995a; e CARVALHO, 1998): Absorção real de $\mathrm{P}=\mathrm{P}$ consumido - (P excretado fezes - $\mathrm{P}$ endógeno).

Na determinação da disponibilidade biológica do $\mathrm{P}$ do fosfato bicálcico, levou-se em conta o fracionamento do fósforo consumido, em partes de origem vegetal, e do fosfato bicálcico, de acordo com a composição das dietas e o percentual de $\mathrm{P}$ dos ingredientes, e do $\mathrm{P}$ absorvido, em partes de origem vegetal, endógena e do fosfato bicálcico. Para a avaliação do fósforo absorvido de origem vegetal nas dietas com fosfato bicálcico, considerou-se o valor da disponibilidade do fósforo, obtido na ração A (sem suplementação de P), de acordo com BELLAVER et al. (1983); para a fração endógena, utilizou-se o valor determinado pela técnica da diluição isotópica, e para o $\mathrm{P}$ absorvido do fosfato bicálcico, deduziu-se do total absorvido os valores encontrados para $\mathrm{P}$ vegetal $\mathrm{e}$ endógeno. A disponibilidade do $\mathrm{P}$ do fosfato bicálcico consistiu, finalmente, da relação entre a quantidade absorvida e consumida do $\mathrm{P}$ do fosfato bicálcico multiplicada por 100.

No final do experimento, os animais foram sacrificados, para retirada de órgãos e tecidos para estudos complementares, e enterrados em local apropriado.

\section{Resultados e Discussão}

Os valores das variáveis estudadas, em função das dietas experimentais, estão apresentados na Tabela 2.

Os valores obtidos para o consumo médio de fósforo apresentaram relação linear significativa com os níveis de $\mathrm{P}$ das dietas, equação $\hat{\mathrm{Y}}=-23,676+389,94 \mathrm{X} ; \mathrm{R}^{2}=0,96$; $\mathrm{P}<0,01$. Este comportamento está em consonância com os dados de MILLER et al. (1964), em pesquisa com suínos recém-desmamados, e os de FERNANDEZ (1995a), com leitões em crescimento, ao observarem aumento na ingestão de fósforo, com o incremento dos níveis deste mineral na dieta, variando de 0,4 a $0,8 \%$ de $\mathrm{P}$.

$\mathrm{A}$ análise dos dados de $\mathrm{P}$ excretado nas fezes e na urina mostrou reposta linear significativa entre o $P$ excretado (Y) e o consumido (X), representada, respectivamente, pelas equações: $\hat{Y}=41,773+$ $0,2338 X, R^{2}=0,60 ; P<0,05$ e $\hat{Y}=-18,067+0,15 X$; $\mathrm{R}^{2}=0,68 ; \mathrm{P}<0,01$. Os resultados estão em concordância com os de VIPPERMAN JR. et al. (1974) e FERNANDEZ (1995b), em que o fósforo nas fezes e na urina cresceu linearmente com os níveis de fósforo na dieta, indicando que a utilização do fósforo consumido é regulada tanto pela ação intestinal como pela renal. Nesse sentido, GEORGIEVSKII (1982) e BREVES e SCHRÖDER (1991) relataram que a excreção de fósforo pela urina funciona como importante mecanismo de controle homeostático do fósforo em não-ruminantes.

$\mathrm{O} \mathrm{P}$ endógeno fecal também aumentou com o incremento do consumo desse mineral, de acordo com a relação $\hat{\mathrm{Y}}=5,8536+0,0226 \mathrm{X} ; \mathrm{R}^{2}=0,40$, $\mathrm{P}<0,01$, o que está de acordo com CHALA e BRAITHWAITE (1988) e CAB (1982), ao destacarem que a excreção endógena não é constante, mas atua como rota de excreção do elemento absorvido em excesso, conforme a necessidade. 
Tabela 2 - Valores das variáveis em função das dietas experimentais

Table 2 - Values of the variables in fuction of the experimental diets

\begin{tabular}{|c|c|c|c|c|c|}
\hline \multirow[b]{2}{*}{ Item } & \multicolumn{5}{|c|}{$\begin{array}{l}\text { Nível de fósforo total (\%) } \\
\text { Level of total phosphorus }\end{array}$} \\
\hline & $\begin{array}{c}\mathrm{A} \\
(0,30)\end{array}$ & $\begin{array}{c}\text { B } \\
(0,40)\end{array}$ & $\begin{array}{c}\mathrm{C} \\
(0,51)\end{array}$ & $\begin{array}{c}\mathrm{D} \\
(0,65)\end{array}$ & $\begin{array}{c}\mathrm{E} \\
(0,73)\end{array}$ \\
\hline Peso inicial & $19,18 \pm 1,03$ & $19,18 \pm 0,88$ & $18,80 \pm 1,00$ & $18,80 \pm 1,04$ & $18,68 \pm 1,07$ \\
\hline $\begin{array}{l}\text { Initial weight } \\
\mathrm{P} \text { consumido } 1,3\end{array}$ & $93,06 \pm 1,02$ & $149,29 \pm 2,95$ & $184,27 \pm 6,16$ & $251,37 \pm 6,89$ & $275,99 \pm 8,61$ \\
\hline $\begin{array}{l}P \text { intake } \\
P \text { fezes } \\
2,3 \\
\text { Fecal } P\end{array}$ & $67,10 \pm 4,97$ & $81,79 \pm 4,24$ & $69,67 \pm 6,78$ & $99,66 \pm 6,13$ & $113,73 \pm 5,91$ \\
\hline $\begin{array}{l}\mathrm{P} \text { urinário } \\
\text { Urinary } P\end{array}$ & $0,28 \pm 0,10$ & $0,46 \pm 0,09$ & $6,31 \pm 2,50$ & $20,14 \pm 7,50$ & $26,39 \pm 1,70$ \\
\hline $\begin{array}{l}\mathrm{P} \text { endógeno fecal }{ }^{1,3} \\
\text { Fecal endogenous } P\end{array}$ & $8,59 \pm 0,88$ & $8,43 \pm 0,23$ & $9,81 \pm 1,07$ & $11,28 \pm 0,81$ & $12,75 \pm 1,55$ \\
\hline $\begin{array}{l}\mathrm{P} \text { retido } 1,3 \\
\text { Retention } P\end{array}$ & $25,68 \pm 5,37$ & $67,05 \pm 4,37$ & $108,29 \pm 6,37$ & $131,56 \pm 0,90$ & $135,88 \pm 14,99$ \\
\hline $\begin{array}{l}\mathrm{P} \text { absorvido }{ }^{1,3} \\
\text { P absorption }\end{array}$ & $34,55 \pm 4,61$ & $75,93 \pm 4,05$ & $124,41 \pm 6,92$ & $162,97 \pm 7,38$ & $175,00 \pm 13,41$ \\
\hline $\begin{array}{l}\text { Coef. absorção } 1,3 \\
\text { Absorption coefficient }\end{array}$ & $0,37 \pm 0,05$ & $0,51 \pm 0,03$ & $0,68 \pm 0,04$ & $0,65 \pm 0,02$ & $0,63 \pm 0,03$ \\
\hline $\begin{array}{l}\text { P plasma }(\mathrm{mg} / 1)^{1,3} \\
\text { Plasm P }\end{array}$ & $72,50 \pm 2,25$ & $77,75 \pm 3,01$ & $86,25 \pm 2,33$ & $84,75 \pm 2,06$ & $79,50 \pm 1,56$ \\
\hline
\end{tabular}

Estes resultados foram similares aos de FERNANDEZ (1995b), ao observar aumento da perda endógena fecal de $\mathrm{P}$ com o incremento do $\mathrm{P}$ consumido, quando o nível ingerido passou de 85,71 para $217,14 \mathrm{mg} \mathrm{P} / \mathrm{kg}$ peso/dia, obtendo para esses padrões de consumo, respectivamente, 9,91 e $11,77 \mathrm{mg}$ $\mathrm{P} / \mathrm{kg}$ peso/dia de perdas endógenas fecais.

Dessa forma, infere-se dos resultados obtidos que a perda endógena fecal representa a resposta fisiológica do animal às várias condições de consumo, e essa perda deve ser então considerada parte integral da avaliação dos alimentos. É importante destacar que, em ensaios de metabolismo sem determinação das perdas endógenas, as variáveis que definem a utilização dos nutrientes apresentam valores subestimados.

Para avaliação das perdas endógenas mínimas, consideraram-se as equações das perdas endógenas fecais e urinárias de $\mathrm{P}$, em função das quantidades consumidas, $\hat{\mathrm{Y}}=5,8536+0,0226 \mathrm{X}$ e $\hat{\mathrm{Y}}=-18,067+0,1509 \mathrm{X}$, com consumo de $\mathrm{P}$ extrapolado para o nível zero. Assim, foram obtidos valores de 5,85 e 18,07 mg P/kg peso/dia, respectivamente, para perdas endógenas fecais eurinárias. O total dessas perdas, $23,92 \mathrm{mg} / \mathrm{kg}$ peso/dia, representa a quantidade mínima de fósforo que o animal deve absorver para suprir as perdas endógenas inevitáveis.

Para converter o valor da perda mínima em exigência de mantença dos leitões, foi encontrado o valor de e $38,58 \mathrm{mg} \mathrm{P} / \mathrm{kg}$ peso/dia, a partir do fósforo consumido em dietas suplementadas com fosfato bicálcico, em que se utilizou o valor médio da eficiência de absorção de fósforo dos tratamentos suplementados com fosfato bicálcico, que foi de $61,66 \%$.

Os valores do $\mathrm{P}$ retido revelam relação linear positiva entre a retenção $(\mathrm{Y})$ e o consumo de $\mathrm{P}(\mathrm{X})$, expressa pela equação: $\hat{Y}=-23,706+0,615 X$; $\mathrm{R}^{2}=0,8876, \mathrm{P}<0,01$, o que está de acordo com o observado por MUDD et al. (1969) e VIPPERMAN JR. et al. (1974), em dietas de leitões em crescimento, com o $P$ variando entre 0,38 e $1,47 \%$ e 0,25 e $0,75 \%$, respectivamente.

A análise da equação $\hat{\mathrm{Y}}=-23,706+0,615 \mathrm{X}$ revela que, ao extrapolar os dados de consumo de $\mathrm{P}$ para o nível zero, os animais estariam em balanço negativo, correspondendo a $-23,71 \mathrm{mg} \mathrm{P} / \mathrm{kg}$ peso/dia. Neste contexto, a retenção de $\mathrm{P}$ será zero, quando o consumo de $P$ for $38,55 \mathrm{mg} / \mathrm{kg}$ peso/dia, o que representa, assim, a quantidade mínima de $\mathrm{P}$ que os animais, nas condições do presente experimento, necessitam. Este valor é praticamente o mesmo obtido, levando em consideração técnica da diluição isotópica para avaliação das perdas endógenas (38,58 mg P/kg peso/dia), o que reforça o balanço de $\mathrm{P}$, como importante indicativo 
na determinação da exigência deste mineral para suínos.

Com relação à absorção de $\mathrm{P}$, observou-se resposta linear positiva com o consumo deste mineral, equação: $\hat{\mathrm{Y}}=-35,925+0,7888 \mathrm{X} ; \mathrm{R}^{2}=0,9472 ; \mathrm{P}<0,01$, o que está de acordo com os resultados observados nos trabalhos de MILLER et al. (1964), VIPPERMAN JR., et al. (1974), BELLAVER et al. (1983) e FERNANDEZ (1995a). Já o coeficiente de absorção do $\mathrm{P}$ apresentou resposta quadrática ao consumo de $\mathrm{P}$ equação: $\hat{\mathrm{Y}}=-0,1068+0,0064 \mathrm{X}-0,000013 \mathrm{X}^{2}$; $\mathrm{R}^{2}=0,70 ; \mathrm{P}<0,01$. Os dados desta variável, expressos em porcentagem, indicam os valores de disponibilidade de $\mathrm{P}$ da dieta.

$\mathrm{Na}$ dieta à base de cereais, o fósforo, além de ter sido menos consumido, refletiu no baixo valor da sua disponibilidade biológica, 37\%, sendo inferior ao encontrado por BELLAVER et al. (1984), em dieta à base de cereais, em que a disponibilidade biológica foi de $66,26 \%$ e o coeficiente de absorção aparente, de 32,56\%.

O comportamento da curva do coeficiente de absorção real mostra que há mecanismo saturável de absorção de $\mathrm{P}$, no nível de consumo de $246,0 \mathrm{mg} \mathrm{P} / \mathrm{kg}$ peso/dia. Como a absorção real teve resposta linear com o consumo de $\mathrm{P}$, pode-se inferir que, em altos níveis de consumo de $\mathrm{P}$, há, também, absorção passiva, provocada pela pressão do $\mathrm{P}$ exercida nos sítios de absorção. Esta constatação está em consonância com BREVES e SCHRÖDER (1991), ao relatarem que, em monogástricos, o transporte de $\mathrm{P}$ no intestino delgado, sítio de maior absorção, consiste de um mecanismo ativo saturável e um passivo não-saturável.

$\mathrm{O}$ valor médio da disponibilidade biológica das dietas com suplementação de $\mathrm{P}$, a partir do fosfato bicálcico, foi de $61,66 \%$, sendo superior aos $45,86 \%$ obtidos por BELLAVER et al. (1983), em dieta de suínos suplementada com fosfato bicálcico.

A disponibilidade biológica média do $\mathrm{P}$ do fosfato bicálcico, quando se eliminou a interferência do $\mathrm{P}$ de origem vegetal, por meio do fracionamento do $\mathrm{P}$ consumido e do absorvido nas frações de origem vegetal, endógena e do fosfato bicálcico, foi de $90,34 \%$, sendo superior aos $75,3 \%$ obtidos por CUPÁK et al. (1972); porém foi inferior aos 101,93\% observados por BELLAVER et al. (1983).

Ao se observar o efeito do $\mathrm{P}$ consumido na concentração de $\mathrm{P}$ no plasma, verificou-se relação quadrática, $\hat{\mathrm{Y}}=44,469+0,3721 \mathrm{X}-0,009 \mathrm{X}^{2}$; $\mathrm{R}^{2}=0,5016 ; \mathrm{P}<0,01$, em que o maior conteúdo de $\mathrm{P}$ foi observado no nível de consumo de $206,7 \mathrm{mg} \mathrm{P} / \mathrm{kg}$ peso/dia.

Para VIPPERMAN JR. et al. (1974), os níveis de P no soro sangüíneo são indicadores pobres do aproveitamento do fósforo, o que pode ser confirmado pelas afirmações de DeLUCA (1979) e GEORGIEVSKII (1982), ao mostrarem que o metabolismo de cálcio e fósforo é, altamente, regulado por um sistema hormonal constituído por paratormônio, calcitonina e metabólitos de vitamina $\mathrm{D}$, que agem no sentido de manter a concentração desses minerais no sangue em níveis suficientes para a adequada mineralização. Dentro desse enfoque, ENGSTRON et al. (1985) relataram que a hipofosfatemia, em dietas deficientes de $\mathrm{P}$ para suínos, se instala progressivamente, atingindo a média mínima de $\mathrm{P}$ inorgânico no plasma, em torno de 34 a 41 dias de restrição do mineral na dieta. Assim, no presente experimento, o período experimental foi insuficiente para manifestação da queda da concentração de $\mathrm{P}$ no plasma, nos tratamentos em que o consumo de $\mathrm{P}$ se apresentou baixo.

No entanto, os resultados obtidos sugerem que o conteúdo de fósforo no plasma pode ser um fator auxiliar na avaliação do status de fósforo em suínos em crescimento, em razão da resposta quadrática manifestada em função do $\mathrm{P}$ consumido. Este comportamento está de acordo com os resultados obtidos por REINHART e MAHAN (1986), trabalhando com níveis de $\mathrm{P}$ dietético com valores entre 5\% abaixo e $10 \%$ acima das exigências, $\mathrm{KOCH}$ e MAHAN (1986), na faixa de 0,09 a $0,31 \% \mathrm{P}$, e GOMES (1988), entre 0,33 e $0,63 \% \mathrm{P}$, ao observarem que a concentração de $\mathrm{P}$ no soro sangüíneo aumentou linearmente em leitões, na fase de crescimento, com o incremento do P nas dietas. A mesma tendência, porém não-significativa, foi constatada por FARMATRE et al. (1977). É importante destacar que os tipos de relações diferentes, linear ou quadrática, podem estar associadas à variação na amplitude de $\mathrm{P}$ consumido e da idade dos animais.

\section{Conclusões}

As perdas endógenas fecais mínimas dos leitões, no início da fase de crescimento $(20 \mathrm{~kg})$, foram de 23,92 $\mathrm{mg} \mathrm{P} / \mathrm{kg}$ de peso/dia.

A exigência de $\mathrm{P}$ para mantença de leitões, em dietas suplementadas com fosfato bicálcico, foi de $38,58 \mathrm{mg} / \mathrm{kg}$ peso/dia.

A disponibilidade biológica do $\mathrm{P}$ do fosfato bicálcico foi de $90,30 \%$.

O conteúdo de $\mathrm{P}$ no plasma foi influenciado pelo $\mathrm{P}$ consumido. 


\section{Referências Bibliográficas}

AKINSOYINN, A.O. 1986. Minimum phosphorus requeriment of the dwarf goat for maintenance. Trop. Agric., 63(4):333335.

ASSOCIATION OF OFFICIAL ANALYTICAL CHEMISTS AOAC. 1980. Official methods of analysis. $13 \mathrm{ed}$. Washington: AOAC. $1018 \mathrm{p}$

BELlAVER, C., GOMES, P.C., FIALHO, E.T. et al. 1984. Absorção e disponibilidade de fósforo de fosfatos naturais em rações para suínos. Pesq. Agropec. Bras., 19(12):1513-1515.

BELLAVER, C., GOMES, P.C., SANTOS, D.L. 1983. Absorção e disponibilidade de fósforo para suínos baseada na diluição isotópica de radiofósforo $\left.{ }^{32} \mathrm{P}\right)$. Pesq. Agropec. Bras., 18(9):1053-57.

BREVES, G., SCHRÖDER, B. 1991. Comparative aspects of gastrointestinal phosphorus metabolism. Nut. Res. Rev., $4: 125-140$

CARVALHO, F.F.R. Efeitos de níveis de fósforo sobre digestibilidade, metabolismo, perda endógena e cinética de fósforo em cabritos saanem. Jaboticabal: FCAV/UNESP, 1998. 83p. Tese (Doutorado) - Faculdade de Ciências Agrárias e Veterinárias, Universidade Estadual Paulista, 1988.

CAVALCANTE, S.G. Biodisponibilidade de fósforo de cinco fosfatos para suínos recém-desmamados e avaliação de seu desempenho produtivo. Belo Horizonte, 1994. 65p. Dissertação (Mestrado) - Universidade Federal de Minas Gerais, 1994.

CHALA, J., BRAITHWAITE, G.D. 1988. Phosphorus and calcium in growing calves with special emphasis on phosphorus homeostasis. I. Studies of the effect of changes in dietary phosphorus intake and calcium metabolism. J. Agric. Sci., 110:573-581.

COMAR, C.L., MONROE, R.A., VISEK, W.J. et al. 1953. Comparison of two isotope methods for determination of endogenous fecal calcium. J. Nut., 50:459-67.

COMMONWEALTH AGRICULTURAL BUREAUX - CAB. The nutrient requirements of ruminant livestock. Farnham Royal: CAB, 1982, p.183-219. Requirements for the major mineral elements: calcium, phosphorus, magnesium, potassium, sodium and chlorine.

CUPÁK, M., PROCHAZKA, Z., JAMBOR, V. 1972. Utilization of phosphorus compounds in pigs after endogenous phosphorus determination by means of ${ }^{32} \mathrm{P}$. Acta Veterinaria Brno, 41:257-262.

DeLUCA, H.F. 1979. The vitamin D system in the regulation of calcium and phosphorus metabolism. Nut. Rev., 37:161-193.

ENGSTRON, G.W., HORST, R.L., REINHART, T.A. et al. 1985. Effect of dietary phosphorus levels on porcine renal 25-hydroxyvitamin D-1 $\alpha$ and 24R-hidroxilase activities and plasma 1,25-dihydroxyvitamin $\mathrm{D}_{3}$ concentratition. J. Anim. Sci., 60(4):1005-1011.

FARMATRE, C.A., MAHAN, D.C., FETTER, A.W. et al. 1977. Effect of dietary protein, calcium and phosphorus levels for growing and finishing swine. J. Anim. Sci., 44(1):65-71.

FERNANDEZ, J.A. 1995a. Calcium and phosphorus metabolism in growing pigs. I. Absorption and balance studies. Livest. Prod. Sci., 41:233-241.

FERNANDEZ, J.A. 1995b. Calcium and phosphorus metabolism in growing pigs. II Simultaneous radio-calcium and radiophosphorus kinetics. Livest. Prod. Sci., 41:243-254.

FISKE, C.H., SUBBARROW, Y., 1925. The colorimetric determination of phosphorus. J. Biol. Chem., 66(2):375-400.

GEORGIEVSKII, V.I. The physiological role of macroelements.
In: GEORGIVIESKII, V.I., ANNENKOV. B.N., SAMOKHIN V.T. Mineral nutrition of animals. 1. ed. London: Butterworths, 1982. p.91-170.

GOMES, P.C. Exigência nutricional de fósforo e sua disponibilidade em alguns alimentos para suinos de diferentes idades. Viçosa, 1988. 163p. Tese (Doutorado em Zootecnia) - Universidade Federal de Viçosa, 1988.

GRACE, N.D., 1981. Phosphorus kinetics in the sheeps. Br. J. Nut., 45:367-374.

INTERNATIONAL ATOMIC ENERGY AGENCY - IAEA. Laboratory training manual on the use of nuclear techniques in animal research. Vienna: IAEA, 1979. 299p. (Technical Report Series, 193).

KOCH, M.E., MAHAN, D.C. 1986. Biological characteristics for assessing low phosphorus intake in finishing swine. $J$. Anim. Sci., 62(1):163-172.

LOFGREEN, G. P. 1960. The availabiliity of the phosphorus in dicalcium phosphate, bone, meal, soft phosphate and calcium phytates for mature wethers. J. Nut., 70(1):58-62.

MILLER, E.R., ULREY, D.E., ZUTAUT, C.L. et al., 1964. Mineral balance studies with the baby pig: Effect of dietary phosphorus level upon calcium and phosphorus balance. $J$. Nut., 82:111-114.

MUDD, A.J., SMITH, W.C., ARMSGTRONG, D.G. 1969. The influence of dietary concentration of calcium and phosphorus on their retention in the body of the growing pig. J. Anim. Sci., 73:189-198.

NASCIMENTO FILHO, V.F., LOBÃO, A.O. 1977. Detecção de P-32 em amostras de origem animal e vegetal por efeito Cerenkov, cintilação líquida e detector GM. Piracicaba: CENA. 25p. (Boletim Científico, 48).

REINHART, G.A., MAHAN, D.C. 1986. Effect of varous calcium:phosphorus ratios at low and high dietary phosphorus for starter, grower and finishing swine. J. Anim. Sci., 63(1):457-466.

ROSTAGNO, H.S., SILVA, D.J., COSTA, P.M.A. et al. 1987. Composição de alimentos e exigências nutricionais de aves e suínos: tabelas brasileiras. Viçosa: Imprensa Universitária. 59p.

SARRUGE, J.R., HAAG, H.P. 1974. Análises químicas em plantas. Piracicaba: ESALQ/USP. 56p.

STATISTICAL ANALYSIS SYSTEM. 1986. SAS System for linear models. Cary: SAS Institute. $211 \mathrm{p}$.

VIPPERMAN JR., P. E., PEO JR., E. R., CUNNINGHAM, P.J. 1974. Effect of dietary calcium and phosphorus level upon calium, phosphorus and nitrogen balance in swine. J. Anim. Sci., 38(4):758-765.

VITTI, D.M.S.S. Avaliação da disponibilidade biológica do fósforo dos fosfatos bicálcico, Patos de Minas, Tapira efinos de Tapira para ovinos pela técnica da diluição isotópica. São Paulo, 1989. 87p. Tese (Doutorado) - Instituto de Pesquisas Energéticas Nucleares, 1989.

ZAGATTO, E.A.G., KRUG, F.J., BERGAMIN FILHO, H. et al. 1979. Merzing in flow injection analysis. Part 2. Determination of calcium, magnesium and posstassium in plant material by flow injection atomic and flame emission spectrometry. Analytica Chimica Acta, 104:279-284. 